\title{
Menck, Peter
}

\section{Ehre, wem Ehre gebührt! Anmerkungen zu einem Text von Britta Behm et al. "Zur Geschichte der Ehrenmitglieder in der DGfE"}

Erziehungswissenschaft 32 (2021) 62, S. 65-69

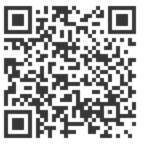

Quellenangabe/ Reference:

Menck, Peter: Ehre, wem Ehre gebührt! Anmerkungen zu einem Text von Britta Behm et al. "Zur

Geschichte der Ehrenmitglieder in der DGfE" - In: Erziehungswissenschaft 32 (2021) 62, S. 65-69 - URN: urn:nbn:de:0111-pedocs-231369 - DOI: 10.25656/01:23136

https://nbn-resolving.org/urn:nbn:de:0111-pedocs-231369

https://doi.org/10.25656/01:23136

in Kooperation mit / in cooperation with:

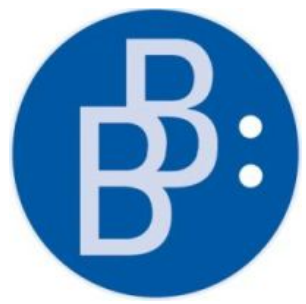

https://www.budrich.de

\section{Nutzungsbedingungen}

Dieses Dokument steht unter folgender Creative Commons-Lizenz: $\mathrm{http}: / /$ creativecommons.org/licenses/by/4.0/deed.de - Sie dürfen das Werk bzw. den Inhalt vervielfältigen, verbreiten und öffentlich zugänglich machen sowie Abwandlungen und Bearbeitungen des Werkes bzw. Inhaltes anfertigen, solange Sie den Namen des Autors/Rechteinhabers in der von ihm festgelegten Weise nennen.

Mit der Verwendung dieses Dokuments erkennen Sie die Nutzungsbedingungen an.

\section{Terms of use}

This document is published under following Creative Commons-License: http://creativecommons.org/licenses/by/4.0/deed.en - You may copy, distribute and render this document accessible, make adaptations of this work or its contents accessible to the public as long as you attribute the work in the manner specified by the author or licensor.

By using this particular document, you accept the above-stated conditions of use.

\section{(c) (7)}

\section{Kontakt / Contact:}

peDOCS

DIPF | Leibniz-Institut für Bildungsforschung und Bildungsinformation Informationszentrum (IZ) Bildung

E-Mail: pedocs@dipf.de

Internet: www.pedocs.de

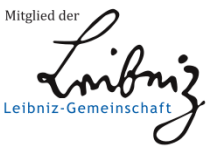




\section{Erziehungswissenschaft}

Mitteilungen der Deutschen Gesellschaft für Erziehungswissenschaft

\section{Heft 62, Jg. 32|2021}

Erziehungswissenschaft und Bildungsrecht Anknüpfungspunkte zur Belebung eines brachliegenden Diskurses

Mit Beiträgen von Hans-Peter Füssel, Martin Heinrich, Julia Hugo, Franz Reimer, Ludwig Salgo, Christine Wiezorek u.a. 


\section{Impressum}

Erziehungswissenschaft

Mitteilungen der Deutschen

Gesellschaft für

Erziehungswissenschaft (DGfE)

Herausgegeben vom Vorstand

der DGfE | www.dgfe.de

ISSN: 0938-5363,

Erscheinungsweise: $2 \mathrm{x}$ jährlich

Online-Ausgabe: https://ew.budrichjournals.de

Herausgebende dieser Ausgabe:

Prof. Dr. Christine Wiezorek, Justus-

Liebig-Universität Gießen

Christine.Wiezorek@erziehung.unigiessen.de

Prof. Dr. Harm Kuper, Freie

Universität Berlin

harm.kuper@fu-berlin.de

Schriftleitung:

Prof. Dr. Bernhard Schmidt-Hertha,

Ludwig-Maximilians-Universität

München

Redaktion und Satz:

Dr. Katja Schmidt, Helmut-SchmidtUniversität/Universität der Bundeswehr Hamburg

Kontakt: ew@dgfe.de

Hinweise für Autorinnen und

Autoren:

www.dgfe.de/zeitschrift-

erziehungswissenschaft

Verlag:

Verlag Barbara Budrich, Opladen, Berlin \& Toronto

www.budrich.de |

www.budrich-journals.de

info@budrich.de

Tel.: (+49) (0)2171 79491 50, Fax:

(+49) (0)2171 7949169
Redaktionsschluss für Heft 63 ist der

15. August 2021

Informationen über die

Mitgliedschaft in der DGfE erhalten

Sie auf der DGfE-Homepage unter

www.dgfe.de/wir-ueber-

uns/mitgliedschaft

oder bei der Geschäftsstelle der

DGfE:

Susan Derdula-Makowski, M.A., Warschauer Straße 36, 10243 Berlin buero@dgfe.de, Tel.: +49(0)30 303 43444, Fax: +49 (0)30 34391853

(C) 2021 Dieses Werk ist bei der Verlag Barbara Budrich GmbH erschienen und steht unter der Creative Commons Lizenz Attribution 4.0 International (CC BY 4.0): https://creativecommons.org/licenses/by/4.0/.

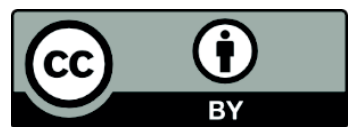

Diese Lizenz erlaubt die Verbreitung, Speicherung, Vervielfältigung und Bearbeitung bei Verwendung der gleichen CC-BY-4.0-Lizenz und unter Angabe der Urheber*innen, Rechte, Änderungen und verwendeten Lizenz.

Dieses Heft steht im Open-AccessBereich der Verlagsseiten zum kostenlosen Download bereit.

Eine kostenpflichtige Druckversion (Print on Demand) kann über den Verlag bezogen werden.

Der Bericht der Sektion 12 - Medienpädagogik, S. 125a-d, ist in der gedruckten Version nicht enthalten. 


\section{INHALTSVERZEICHNIS}

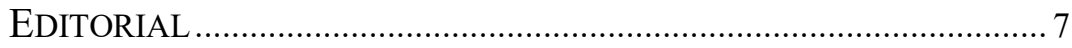

THEMENSCHWERPUNKT

„ERZIEHUNGSWISSENSCHAFT UND BILDUNGSRECHT ANKNÜPFUNGSPUNKTE ZUR BELEBUNG EINES BRACHLIEGENDEN DISKURSES“

Julia Hugo \& Martin Heinrich

$\mathrm{Zu}$ den Eigenlogiken rechts- und erziehungswissenschaftlicher

Perspektiven auf Bildung - die Kommission Bildungsorganisation, Bildungsplanung und Bildungsrecht (KBBB) auf der Suche nach ihrem dritten „B“

Christine Wiezorek \& Franz Reimer

Der Integrationsauftrag der Schule - erziehungs- und rechtswissenschaftliche Perspektiven.

Hans-Peter Füssel

Chancengleichheit im Bildungswesen - einige Überlegungen aus rechtlicher Sicht. Zugleich ein Beitrag zum Zusammenwirken von Rechts- und Erziehungswissenschaft

Ludwig Salgo

(Wozu) Brauchen Pädagog*innen Rechtskenntnisse?

Ein Zwischenruf .... 47

\section{ALLGEMEINE BEITRÄGE}

Peter Menck

Ehre, wem Ehre gebührt! Anmerkungen zu einem Text von Britta

Behm et al. ,Zur Geschichte der Ehrenmitglieder in der DGfE“ 65 


\section{MiTTEILUNGEN DES VORSTANDS}

Protokoll der Mitgliederversammlung der Deutschen Gesellschaft für Erziehungswissenschaft e. $V$................................................................... 71

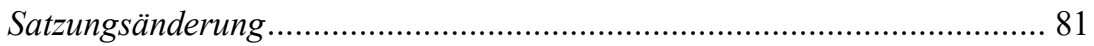

Zur Satzungsänderung und den Verfahrensregeln, die Vergabe des ErnstChristian-Trapp-Preises betreffend ....................................................... 83

Bericht des Wahlausschusses zu den Wahlen des Vorsitzenden und der turnusmäßig zu wählenden Vorstandsmitglieder der DGfE ..................... 87

Ent|grenz|ungen | 28. DGfE-Kongress 2022 - Call for Papers .................. 89

\section{BERICHTE AUS DEN SEKTIONEN}

Sektion 1 - Historische Bildungsforschung ........................................... 95

Sektion 2 - Allgemeine Erziehungswissenschaft .................................... 98

Sektion 3 - Interkulturelle und International Vergleichende

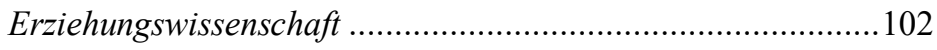

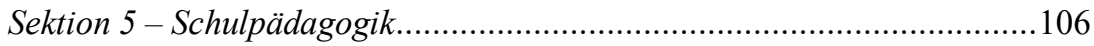

Sektion 6 - Sonderpädagogik ...........................................................111

Sektion 7 - Berufs- und Wirtschaftspädagogik .......................................113

Sektion 8 - Sozialpädagogik und Pädagogik der frühen Kindheit ..............116

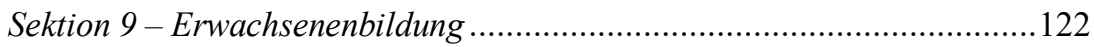

Sektion 11 - Frauen- und Geschlechterforschung ...................................124

Sektion 12-Medienpädagogik..................................................... 125a

Sektion 14 - Organisationspädagogik ................................................126

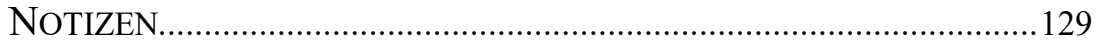




\section{PERSONALIA}

Nachruf auf Prof. em. Dr. Dr. h. c. mult. Oskar Anweiler ............................137

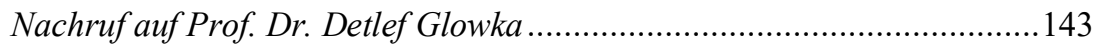

Nachruf auf Prof. Dr. Dr. h. c. mult. Hans-Uwe Otto ..................................145 


\title{
ALLGEMEINE BeITRÄGE
}

\section{Ehre, wem Ehre gebührt!}

Anmerkungen zu einem Text von Britta Behm et al. „Zur Geschichte der Ehrenmitglieder in der DGfE“ ${ }^{\star 1}$

\author{
Peter Menck
}

Der Vorstand der DGfE habe im Februar 2017 ,,[eine] Anfrage zu den Aktivitäten eines Ehrenmitglieds der DGfE im Nationalsozialismus [...] zum Anlass genommen, eine Überprüfung sämtlicher Ehrenmitglieder in Erwägung zu ziehen. Als erster Schritt zu diesem Ziel wurde eine historisch arbeitende Pilotgruppe [...] eingerichtet ${ }^{62}$, so teilte er mit. Diese Pilotgruppe sollte also so etwas wie eine Machbarkeitsstudie erstellen. Sie hat gearbeitet und dem Vorstand „einen vorläufigen Bericht zur internen Information“ vorgelegt; eine ,stark gekürzt[e]“ (S. 78) Zusammenfassung wurde in der Erziehungswissenschaft abgedruckt.

Ich wurde vehement, wie vermutlich die meisten Erziehungswissenschaftler meiner Generation, an Auseinandersetzungen in den 1980er Jahren erinnert, als nämlich der damalige Vorstand Theodor Wilhelm die Ehrenmitgliedschaft verliehen hatte (oder wollte er es erst?). Sie waren der Ausgangspunkt für eine Vielzahl von Untersuchungen zur sogenannten „NS-Vergangenheit“ von Personen, die in Pädagogik und Erziehungswissenschaft als Referenzen in Anspruch genommen wurden und werden. ${ }^{3}$

Bei der Lektüre des Beitrages ist mir als erstes aufgefallen, dass die Pilotgruppe auf diese Vorarbeiten nicht zurückgegriffen, sie jedenfalls nicht berücksichtigt hat. ${ }^{4}$ Das lässt vermuten, dass sie ihren Auftrag inhaltlich und methodisch anders verstand als einen zur Anfertigung einer Machbarkeitsstudie, vielmehr als einen zur Suche nach einem neuen Zugang zu dem alten Thema. Tatsächlich hatte der Vorstand auch eine neue Perspektive vorgegeben: Es sei

1 In: Erziehungswissenschaft. Mitteilungen der Deutschen Gesellschaft für Erziehungswissenschaft 60, 31, 2020, S. 77-100. Im Folgenden weise ich Zitate aus diesem Text durch Angabe der Seitenzahl nach.

2 https://www.dgfe.de/dgfe-wir-ueber-uns/vorstandskommissionen [Zugriff: 18. Juli 2020].

3 Alexander Hesse (1995) hat - nicht nur punktuell wie damals und offensichtlich jetzt bei den Ehrenmitgliedern einer wissenschaftlichen Gesellschaft ansetzend - die Biographien einer Gesamtheit rekonstruiert. In diesem Korpus sind immerhin acht unserer Ehrenmitglieder vertreten (Asmus, Bohnenkamp, Flitner, Geißler, Hetzer, Kittel, Lochner, Seidelmann).

4 Das 78 Titel umfassende Literaturverzeichnis enthält nur 30 Titel, die vor 2010 erschienen sind; davon haben nur sechs einen ersichtlichen, thematischen Bezug zum Thema der Studie. 
„,zu ermitteln, wie umfangreich die Überprüfung der bislang 54 Ehrenmitglieder in ihren vielfältigen Verflechtungen ausfallen müsste", also nicht nur derjenigen Ehrenmitglieder, die sich tatsächlich oder möglicherweise zu sehr auf den Nationalsozialismus bzw. die von ihm beherrschten staatlichen Institutionen eingelassen haben - nein aller, demnach auch der drei letzten, die das ihres Alters wegen gleich gar nicht gekonnt haben. ${ }^{5}$ Neu ist an dem Auftrag zudem, dass die zu überprüfenden Verflechtungen nicht näher spezifiziert sind, sich demnach also nicht unbedingt allein auf NS-Verstrickungen beschränken müssen. Die Pilotgruppe hat zwar nicht ausdrücklich mitgeteilt, wie sie diesen Auftrag interpretiert hat. Aber das lässt sich aus dem Beitrag rekonstruieren.

Zunächst einmal will man offensichtlich suchen, dabei Verweisen folgen, Ungeklärtes und Fragen überprüfen, ermitteln. Man denkt an umfangreiche, vergleichende Archivforschungen, ,eine internationale Archiv- und breit angelegte Quellenrecherche“ - kurz zusammengefasst in sieben Punkten am Ende. ${ }^{6}$ Das ist nicht wenig und das ist aufwendig; darauf wird auch hingewiesen. Aber das ist noch nicht einmal alles.

Das Literaturverzeichnis mit seinen vorwiegend jüngeren Titeln legt die Vermutung nahe, dass die Projektgruppe in erster Linie daran interessiert ist, sozusagen im Vorfeld die unterschiedlichsten methodischen Zugänge daraufhin zu überprüfen, was sie für die Interpretation der zu erhebenden Daten leisten könnten: Theorien, Ansätze, Zugänge, Perspektiven sollen offensichtlich mithilfe von Beobachtungen, Spurensuche, der Sichtung von Vorhandenem usw. zur Eingrenzung dessen führen, was der Vorstand mit seinem vergleichsweise offenen Auftrag verband.

Es sieht also so aus, dass die Gruppe ihren Auftrag nicht als eine Machbarkeitsstudie verstanden hat. Nicht wird ein bestimmtes Forschungsprojekt auf seine Machbarkeit hin untersucht, vielmehr wird zusammengestellt, welche Randbedingungen erfüllt sein müssten, damit ein solches überhaupt erst konzipiert werden kann. Wenn meine Vermutung zuträfe, wäre das Ergebnis enttäuschend. Denn nehme ich das alles zusammen - die Menge der zu ermittelnden Daten, dazu die große Zahl der Ansätze zu deren Interpretation, so habe ich den Eindruck, dass das Ganze auf ein viel zu umfangreiches Projekt hinausliefe, als dass es von einer Arbeitsgruppe der üblichen Größe bewältigt werden könnte.

Aber ich kann mich auch täuschen:

5 Birgit Herz und Heinz-Hermann Krüger; auch Hans Merkens, der 1945 noch etwas zu jung für die zu rekonstruierenden „vielfältigen Verflechtungen“ war, die der Vorstand im Auge gehabt haben mag. - Auch das wurde damals, sehr polemisch, diskutiert, ob sich etwa bei den Jüngeren noch braunes Gedankengut finde.

6 Nicht nur am Ende - der Text ist über weite Strecken eine durch auch, etwa, zudem markierte Aneinanderreihung (an die 20 habe ich gezählt) von Sachen, die man, eben, machen könnte. 
„Die hier angerissenen Fragen könnten aber auch sinnvoll mit breiter Beteiligung auf einem Workshop erörtert, ergänzt oder modifiziert werden. Diese Form der (fach-)öffentlichen wissenschaftlichen Auseinandersetzung hat sich bei anderen Fachgesellschaften bewährt". (S. 86)

Dieser Vorschlag ganz am Ende hat mich einigermaßen überrascht. Bekanntlich sind „Workshops“ eher Foren für die Präsentation von Projekten oder Arbeitsergebnissen, vielleicht auch für die Präzisierung von ,,angerissenen Fragen“. Aber für die Ausarbeitung eines bestimmten Vorhabens, hier einer Machbarkeitsstudie, sind sie eher ungeeignet.

Als Beispiel mag ein von einem früheren Vorstand angeregter Workshop zu den sogenannten „Missbrauchsfällen“ dienen. Der Bericht, den Werner Thole u. a. (2012) vorgelegt haben, versammelt eine Reihe von aufschlussreichen Daten und Interpretationsansätzen, zeigt aber nicht, dass da tatsächlich das Problem von Macht und Pädagogik der Erziehungswissenschaft als Wissenschaft zur Bearbeitung, gar bearbeitbar auf den Tisch gelegt wurde. Jedenfalls passt der Umfang der von den Autorinnen und Autoren ins Auge gefassten Arbeiten nicht mit dem vergleichsweise kleinen Format eines Workshops zusammen.

Zurück zum Thema Ehrenmitgliedschaft in einer Standesvertretung der Erziehungswissenschaft. Man kann der Meinung sein, dass eine Erhebung der im weitesten Sinne biographischen Daten der Ehrenmitglieder als solche interessant und lehrreich ist. Ich teile diese Meinung nicht. Für die Erziehungswissenschaft sind solche Daten erst dann und nur insoweit lehrreich, wie sie ersichtlich Antworten auf eine Frage geben, die für die Arbeit der Erziehungswissenschaft relevant ist. Soweit ich das sehe, hieße das zunächst einmal, dass man den Umfang des zu erhebenden Materials sehr viel enger eingrenzen müsste. Und es hieße vor allem, dass man andererseits die moralischen Implikationen, die Bedeutung der Daten für die Erziehungswissenschaft sehr gründlich untersuchen müsste, die ein Interesse an der Biographie einzelner sowie die methodische Suche nach Zugängen zu komplexen und umfangreichen Daten anleiten könnten.

Bei der Auswahl des Materials sollte man in Rechnung stellen, dass es sich in unserem Zusammenhang um die Wissenschaft von Erziehung handelt. Biographische und bibliographische Daten, Dokumente also von Tätigkeit und Schriften der Geehrten, sind m. E. nur so weit von Belang, wie sie ersichtlich über Erziehungswissenschaft und ihren Gegenstand Auskunft geben. Was die im Übrigen gesagt, getan, gar gedacht haben, ist - noch einmal - für die Erziehungswissenschaft und für die Erziehungswissenschaft nicht relevant. ${ }^{7}$ Gleichwohl dürfte auch bei einer solchen Beschränkung immer noch eine Menge an Arbeit übrig bleiben. Aber bekanntlich ist in dieser Hinsicht in den vergange-

7 Dass Hildegard Hetzer zum Beispiel ihre „ehemalige Arbeitskollegin und Vorgesetzte“ in ein und derselben Quelle einmal als „Halbjüdin, ein andermal als ,die bestangezogene Frau von Wien“ bezeichnet habe, ist doch wohl eher fürs Feuilleton als für die Wissenschaft von Belang (80). 
nen Jahrzehnten sehr viel eruiert worden. Darauf könnte man zurückgreifen. Dem Beitrag kann ich nicht entnehmen, dass die Gruppe so etwas beabsichtigt.

Schwieriger ist das mit der moralischen Seite der Sache, wie ich das abkürzend genannt habe. Noch einmal der Arbeitsauftrag: eine ,,zu ermitteln, wie umfangreich die Überprüfung der [...] 54 Ehrenmitglieder in ihren vielfältigen Verflechtungen ausfallen müsste“8. Diese Formulierung lässt völlig offen, an was für Verflechtungen man dabei gedacht haben mag. Zwar lassen Auftraggeber und jedenfalls die Auftragnehmer wenig Zweifel daran, dass sie an diejenigen gedacht haben, die sich möglicherweise durch - und hier kommt die Moral ins Spiel - ihre Nähe zur NS-Ideologie und -Praxis kompromittiert haben. Wenn das so sein sollte, teilte man eine Prämisse, die auch den meisten der erwähnten älteren Arbeiten zugrunde liegt, kurz gesagt: Wir und die Anderen; Wir - das sind die Unbelasteten; die Anderen - die in unserer Zunft eigentlich nicht am Platze sind, jedenfalls nicht als Ehrenmitglieder. ${ }^{9}$ Diese Prämisse halte ich für durchaus unbrauchbar, wenn man sie einer Suche nach dem Selbstverständnis einer Wissenschaft zugrunde legt, in unserem Falle der Erziehungswissenschaft.

Fazit: Der Vorstand hat aus nicht näher erläuterten Gründen eine nicht näher erläuterte, anonyme Anfrage nicht alsbald beantwortet, vielmehr eine Arbeitsgruppe damit betraut, die erforderlichen Vorarbeiten für eine umfassende Antwort zu leisten. Dieses Verfahren ist durchaus nicht unüblich. Der Auftrag des Vorstands ist allerdings so vage, dass er auf sehr unterschiedliche Weise interpretiert werden kann. Die Arbeitsgruppe legt jetzt nicht, wie man erwarten könnte, ein machbares Arbeitsvorhaben vor. Stattdessen stellt sie zusammen, was alles vorweg geleistet werden müsste, damit ein sinnvolles und bearbeitbares Forschungsvorhaben überhaupt erst geplant werden könnte.

Für das Selbstverständnis der Erziehungswissenschaft ist das Sammeln von Daten und Interpretationsansätzen unergiebig. Für ertragreicher halte ich es, wenn man Fragen wie diese zu beantworten versuchte:

Welches sind die Randbedingungen (im weitesten Sinne), die gegeben sein müssen, damit Einzelne, die in pädagogischen Einrichtungen arbeiten, nicht deren leitenden Normen widersprechend handeln?

Welches sind die Randbedingungen (im weitesten Sinne), die gegeben sind, wo Einzelne nicht pädagogisch legitim gehandelt haben oder handeln?

Vielleicht präzisiert der Vorstand auf der Basis des Berichts der Arbeitsgruppe seinen Auftrag in diesem Sinne oder auch in eine andere Richtung.

Peter Menck, Prof. Dr., ist emeritierter Universitätsprofessor für Erziehungswissenschaft an der Universität Gießen.

8 https://www.dgfe.de/dgfe-wir-ueber-uns/vorstandskommissionen [Zugriff: 18.Juli 2020]

9 Diese Einschränkung ist nötig, denn andernfalls müsste man ziemlich viele ausschließen. 


\section{Literatur}

Behm, Britta/Grube, Norbert/Hoffmann-Ocon, Andreas/Rohstock, Anne (2020): Zur Geschichte der Ehrenmitglieder in der DGfE. Auszüge aus dem Zwischenbericht der bildungshistorischen Pilotgruppe. In: Erziehungswissenschaft. Mitteilungen der Deutschen Gesellschaft für Erziehungswissenschaft 31, 60, S. 77-100. https://doi.org/10.3224/ezw.v31i1.09.

Hesse, Alexander (1995): Die Professoren und Dozenten der preußischen Akademien (1926-1933) und Hochschulen für Lehrerbildung (1933-1941). Weinheim: Deutscher Studien Verlag.

Thole, Werner et al. (Hrsg.) (2012): Sexualisierte Gewalt, Macht und Pädagogik. Opladen: Budrich. https://doi.org/10.2307/j.ctvdf0b6p. 